\title{
Breast sarcoma surgical management: a five-year multicentric study
}

\author{
Răzvan V. Scăunașu ${ }^{1}$, Traean Burcoș ${ }^{1}$, Ștefan Voiculescu ${ }^{1}$, Cristina Cozma ${ }^{1}$, Bogdan \\ Popescu $^{1}$, Eugen Brătucu ${ }^{2}$ \\ ${ }^{1}$ Carol Davila University, Department of General Surgery, Colțea Clinical Hospital, Bucharest, Romania \\ ${ }^{2}$ Carol Davila University, Department of General Surgery, Prof. Dr. Al. Trestioreanu, Oncology Institute, \\ Bucharest, Romania
}

\begin{abstract}
Background. Breast sarcomas are a heterogeneous group of breast neoplasms with a low incidence and a reserved prognosis. No treatment protocol has been yet established, a guideline similar to soft tissue sarcomas is used.

Materials and Methods. Our study analyzed all the patients admitted with the diagnosis of malignant breast disease in two specialized centers on a five-year time frame. We compared long term results for the patients who underwent conservative treatment and mastectomy.

Results. A total of 76 cases received surgical treatment with curative intent, 24 conservative procedures and 52 mastectomies. Incidence of local recurrence does not appear to be closely related to the type of surgical procedure. There were a number of five local recurrences for patients who received conservative treatment and 7 local recurrences where we used mastectomy. Kaplan-Meier analysis conducted shows no differences statistically significant $(\operatorname{sig}=0.459)$ between the results of conservative treatment and mastectomy. Basically conservative surgery seems to get similar results, provided that $\mathrm{R}_{0}$ resection objective can be met.

Conclusions. Treatment options are more limited for breast sarcomas than carcinomas, the role of surgery being more important to therapeutic success. The biological characteristic of the tumor including histological type and sub-type, play an important role in determining the results and the treatment should be tailored and adapted for each case.
\end{abstract}

Keywords: breast sarcoma, non-epithelial cancer, surgical management 


\section{Introduction}

Breast sarcomas are a heterogeneous group of mammary tumors developed from mesenchymal tissue. These are encountered in practice with a reduced frequency. The literature reports that breast sarcomas represent approximately $5 \%$ of all soft tissue sarcomas and about $1 \%$ of breast neoplasia (1). Even though they show some similarities with adenocarcinomas and have a low incidence, sarcomas have an important place in neoplastic breast pathology due to the reserved prognosis.

Better outcomes can occur by recognizing the clinical and evolutionary particularities associated with the adaptation of diagnostic and treatment protocols. Unfortunately, due to the low incidence, this pathology is not well documented, literature highlighting only studies with a small number of subjects or extended over many years (which does not allow a comparative analysis). Also, there is no multicenter, randomized study that could establish a treatment protocol or consensus, so nowadays the therapeutic conduct is extrapolated from the treatment guidelines used for soft tissue sarcomas.

The clinical examination of breast sarcomas unveils a tumoral mass with increased consistency, relatively well defined, which, in early stages can be easily considered benign. Rapid growth and patient's age are the only elements of concern. In advanced stages, clinical manifestations betray the neoplastic character, producing deformities and significant asymmetry of the breasts, through chest wall infiltration and attachment, skin changes which may go up to ulceration and bleeding or developing a significant collateral circulation.

\section{Materials and Methods}

Our study analyzed all the patients admitted with the diagnosis of malignant breast disease in Oncological Institute "Prof. Dr. Alexander Trestioreanu" Bucharest and" Colţea" Clinical
Hospital during 2011 to 2015 (5 years). This analysis was possible with the permission and written consent of the two institutions; I thank them here.

The study has a retrospective character; the timeframe of five years being chosen to comprise a large enough group of patients for obtaining statistically significant results.

For creating the groups of patients were queried databases used for reporting in the DRG system (Diagnosis Related Groups). The classification system is, as its name implies, a classification scheme of the patients based on the diagnosis. This system is similar to the international disease classification system (International Classification of Diseases - ICD), in which the diagnoses are classified into classes and subclasses (2).

Repeated hospitalizations were queried with diagnoses and procedures performed on these occasions. These tools are extremely useful for tracking and monitoring patients, with a given limitation, that they must be admitted in the same medical institution.

Exclusion criteria: cases whose data break technical specifications of the minimum data patient set and cases where we could not register the result of the histopathological examination.

For this study, we classified sarcomas following histological criteria, according to WHO recommendations (3) and to the European Society of Medical Oncology (ESMO) (4). Thus, cases of malignant Phyllodes tumor were excluded and analyzed separately.

Breast surgery procedures codes where divided into two classes. Conservative surgery includes procedures like sectorectomy, quadranectomy or atypical oncoplastic resections which partially preserve the breast. Also, mastectomies contain variations that preserve the skin or the nipple-areolar complex (skin sparing, nipple sparing), but with total excision of the mammary gland. 


\section{Results}

Analysis of the histopathological results identified a number of 102 cases classified as primary and secondary breast sarcomas.

A total of 88 cases received surgical treatment, 24 conservative procedures and 64 mastectomies. A number of 12 surgeries were performed for advanced stages with palliative intent, due to local complications (bleeding ulcers, infection, and necrosis).

With exception of an immediate reintervention for hemostasis, postoperative complications were rare, had a favorable outcome with conservative treatment. There was no intra-operative or in-hospital mortality for this group. There were no cases of arm lymphedema after surgery.

It should be noted that these results are obtained from centers with a large number of patients and with a rich experience in the treatment of breast tumor pathology, surgical procedures performed being considered routine.

\section{Follow-up and studying late complications}

Accurate data considering follow-up and patient's evolution is extremely difficult to collect in Romania, in the absence of institutionally sustained programs and empowered follow-up protocols to facilitate data recording, increase the compliance and facilitate communication with patients.

Among the data that has been collected, we considered local recurrence as the most reliable and important element. Local recurrence is mentioned upon repeated hospitalizations in the study time-frame (5 years). There were excluded 12 cases for which the initial surgery had a palliative intent. If the histopathological result of the initial tumor was not sarcoma, the cases were not considered relapses.

Reporting the number of patients with local recurrence (12) to the number of patients who benefited from surgery with curative intent (76 interventions) we obtain a rate of $15.78 \%$. The literature records a more severe prognosis of breast sarcomas, compared to carcinomas, the rates of local recurrence mentioned in similar studies sometimes reaching $34 \%$ (5-8). It is difficult to say whether the good results we obtained are due to aggressive surgical attitude (manifested by a comparatively higher number of performed mastectomies), time limited interval of the study (local recurrence can occur later) or loss of cases from study (cases that were admitted in other medical institutions).

Incidence of local recurrence does not appear to be closely related to the type of surgical procedure. There were a number of five local recurrences for patients who received conservative treatment and 7 local recurrences where we used mastectomy. Kaplan-Meier analysis conducted shows no statistically significant differences ( $\mathrm{Sig}=0.459$ ) between the results of conservative treatment and mastectomy (Figure 1). Basically conservative surgery seems to get similar results, provided that $\mathrm{R} 0$ resection objective can be met.

\section{Discussions}

Surgery is the standard treatment of all breast malignancies in the early stages. Considering breast sarcomas, due to modest results of neoadjuvant therapy, the role of surgery is even more important for therapeutic success. Surgical procedures have constantly evolved along history and today there are numerous surgical techniques used for breast neoplastic pathology, but all techniques pursue the same goal: total excision of tumoral tissue, with a safety margin of at least $1 \mathrm{~cm}$ of healthy tissue. It is considered the most important predictive factor of the therapy outcome for sarcomas (1).

Conservative treatment is a viable and desired alternative to mastectomy only if similar oncologic results can be obtained and the remaining tissue allows for a good aesthetic result. It is not always applicable, even in the early stages depending on the patient's conformation and consent. In practice, there is often a situation where tumor volume/glandular volume report is unfavorable, but also one where the patient opts for 
mastectomy, and even more, for a bilateral mastectomy unjustified by the situation. Regarding sarcomas, there is no benefit of a contralateral prophylactic mastectomy.

The surgical technique followed the indications and contra-indications for each procedure.

Absolute contra-indications for conservative breast surgery $(9,10)$ :

- persistent positive margins after conservative surgery

- multi-focal involvement (quite exceptional in sarcomas)

- history of prior radiation in the treatment area

- inability to undergo radiation therapy for invasive disease

- first or second trimester of pregnancy

Relative contra-indications of the procedure include $(11,12)$ :

- low glandular volume

- large tumor

- $\quad$ node metastasis

- collagen or vascular disease

Patients and surgeons alike want to obtain a good aesthetic result. Oncologic considerations require, besides excision of the entire tumor mass, obtaining $\mathbf{R}_{0}$ safety margins with no microscopically detectable invasion. This excision depends on many factors such as tumor size compared to available glandular volume, tumor location and distance to adjacent anatomical structures, often making it impossible to avoid mastectomy. Histopathological sub-type also can require larger than standard safety margins, as is the case for angiosarcoma, where $1 \mathrm{~cm}$ may be insufficient. Where after repeated attempts a safety margin cannot be obtained, mastectomy is advised. All these arguments have led to an increased number of mastectomies in our group.

Breast cancer treatment is multi-modal and realized in multi-disciplinary teams, which should not exclude the radiologist, surgeon, pathologist,
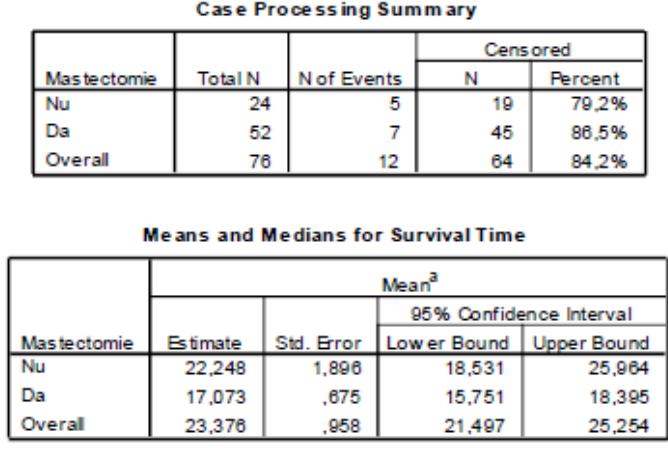

a. Estimation is limited to the largest survival time if it is censored.
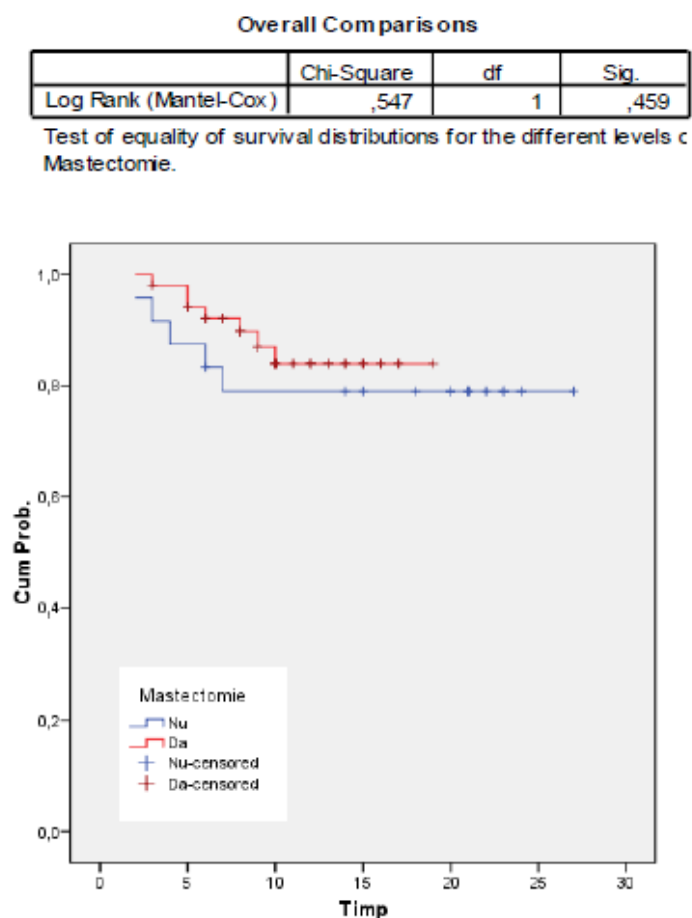

Figure 1. Kaplan-Meier analysis of mastectomy and conservative surgical procedures for local recurrence

oncologist, radiotherapist, and plastic surgeon or psychologist. The qualifications, expertise and cooperation of these members realize exceptional results that can be obtained in the treatment of breast neoplasm. To facilitate this communication and cooperation between specialists, a regularly meeting of the oncology committees can be useful to discuss the difficult cases. International guidelines recommend in addition creating centers of breast treatment able to focus a minimum of 150 cases treated per year (13, 14). The cases from this study were taken from two centers (Oncological Institute Bucharest and Colțea Clinical Hospital) that achieve this objective. 
The most commonly used therapeutic method in our study was surgical treatment. The therapeutic algorithm of sarcomas in general and in particular of those localized in breast, surgery is considered the "first violin" in the orchestra of therapeutic means. The purpose of the various types of surgical treatments is to obtain a full excision of the tumor tissue with microscopically tumor free margins $\left(\mathrm{R}_{0}\right)$. This is considered the most important predictive factor in sarcoma therapy (1).

The main causes that restrain performing surgical treatment relate to the advanced stage of the disease, patients condition or sometimes their refusal.

There are no established protocols of radiotherapy for breast sarcomas. The indication of adjuvant radiotherapy is taken from the protocols for soft tissue sarcomas, where favorable results were obtained using a radiation dose of 50 Gy to the breast and at least 60 Gy to the tumor bed area. Using this protocol, McGowan and collaborators have achieved a decrease in relapse rates from $34 \%$ to $13 \%$ and an improvement in 5 years survival from $50 \%$ to $91 \%$ (15-17).

Chemotherapy has modest results and is a method reserved for local advanced stages. Whenever possible, the surgical treatment of lung or liver metastasis is preferred, and subsequently associated with polichemotheraphy. This therapy, use as first-line treatment anthracycline with or without doxorubicin or ifosfamide (11). There are no randomized studies that certify the supremacy of certain combinations.

Unfortunately, treatment options are more limited for sarcomas comparing with carcinoma: hormone therapy is unnecessary and biological therapy is in the experimental stage. Initiated studies scan the results of such agents, like Sunitinib and Crizotinib, but their indication is still unclear (18-21). For angiosarcomas favorable results could be obtained from anti- angiogenic or anti-endothelial growth factor treatments (sorafenib, bevacizumab), but currently, these are in trial phase and are not included in our treatment guidelines.

\section{Conclusions}

The biological characteristics and evolution patterns of the primary tumor play an important role in determining the results. Even before the emergence of molecular profiling techniques, pathologists recognized that breast cancer was heterogeneous through morphological observations.

Each pathological type shows a unique clinical profile in terms of presentation, prognosis or available treatment options. The individualized surgical attitude should improve the morbidity, along with psychological and aesthetic benefits. We consider that the therapeutic management of breast tumors should be customized depending on the disease and the patient.

The patient is the most important decision factor. The management plan depends on his tolerance of the proposed treatment, genetic vulnerability, consent or preference.

The disease affects the therapeutic decision by a number of factors such as tumor size and location, the existence of multiple multi-centric or multi-focal lesions, local invasion or regional and distant metastasis.

Another element that is not yet fully elucidated, but which we consider of great importance is the biology of the tumor. Histopathological types of the tumor, the presence or absence of expression of certain receptors or genes create the tumor profile and the treatment should be created and adapted for each case. Undoubtedly, technological advances will facilitate the growth of knowledge derived from bio-molecular and genetic study of this pathology will enable detailed characterization of tumors and will discover new treatment options. 


\section{References}

1. Yin M, Mackley HB, Drabick JJ, Harvey HA. Primary female breast sarcoma: clinicopathological features, treatment and prognosis. Sci Rep. 2016; 6: 31497. PMID: 27510467 https://doi.org/10.1038/srep31497

2. Ramalho I, Campos S, Rebelo T, Figueiredo Dias M. A Scary Onset of a Rare and Aggressive Type of Primary Breast Sarcoma: A Case Report. Case Rep Oncol. 2016; 9(3): 796-801. PMID: 28101028 https://doi.org/10.1159/000452946

3. Castro-González E, Bastida J, Rivero-Vera JC, Camacho-Galán R, Limeres-González MA, Jiménez-Bravo de Laguna S, Valerón-Almazán P. Fletcher Cutaneous and breast Langerhans cell sarcoma. J Eur Acad Dermatol Venereol. 2016; 30(10): e33-e34. PMID: 26332733

https://doi.org/10.1111/jdv.13282

4. Lázár G, Bursics A, Farsang Z, Harsányi L, Kósa C, Maráz R, Mátrai Z, Paszt A, Pavlovics G, Tamás R. Modern surgical treatment of breast cancer. 3rd Breast Cancer Consensus Conference. Magy Seb. 2016; 69(3): 117-32.

\section{https://doi.org/10.1556/1046.69.2016.3.5}

5. Pencavel TD, Hayes A. Breast sarcoma - a review of diagnosis and management. Int J Surg. 2009; 7(1): 20-3. PMID: 19114317

https://doi.org/10.1016/j.ijsu.2008.12.005

6. Adem C, Reynolds C, Ingle JN, Nascimento AG. Primary breast sarcoma: clinicopathologic series from the Mayo Clinic and review of the literature. Br J Cancer 2004: 91(2): 237-41. PMID: 15187996 https://doi.org/10.1038/sj.bjc.6601920

7. Kondi-Pafiti A, Dellaportas D, Myoteri D, Tsagkas A, Ntakomyti E, Kairi-Vasilatou E. Rare non-epithelial primary breast neoplasms: a tenyear experience at a Greek University Hospital. $J$ BUON. 2013; 18(1): 70-6. PMID: 23613391
8. O'Donnell ME, McCavert M, Carson J, Mullan FJ, Whiteside MW, Garstin WI. Non-epithelial malignancies and metastatic tumours of the breast. Ulster Med J. 2009; 78(2): 105. PMID: 19568446

9. Hsu C, McCloskey SA, Peddi PF. Management of Breast Sarcoma. Surg Clin North Am. 2016; 96(5): 1047-58. PMID: 27542642

\section{https://doi.org/10.1016/j.suc.2016.05.004}

10. Vallonthaiel AG, Kaushal S, Gogia A, Mathur SR. Bilateral Myeloid Sarcoma of Breast in a Young Male Occurring after 11 Years of Chronic Myeloid Leukemia Diagnosis: A Unique Pattern of Relapse. Breast J. 2016; 22(6): 692-693. PMID: 27488267 https://doi.org/10.1111/tbj.12655

11. Johnson ED, Gulbahce E, McNally J, Buys SS. Malignant Phyllodes Tumor Presenting in Bone, Brain, Lungs, and Lymph Nodes. Case Rep Oncol. 2016; 9(3): 861-868. PMID: 28203179 https://doi.org/10.1159/000453660

12. McGowan TS, Cummings BJ, O’Sullivan B, Catton CN, Miller N, Panzarella T. An analysis of 78 breast sarcoma patients without distant metastases at presentation. Int J Radiat Oncol Biol Phys. 2000; 46(2): 383-90. PMID: 10661345 https://doi.org/10.1016/S0360-3016(99)00444-7

13. Sciubba DM, Goodwin CR, Yurter A, Ju D, Gokaslan ZL, Fisher C, Rhines LD, Fehlings MG, Fourney DR, Mendel E, Laufer I, Bettegowda C, Patel SR, Rampersaud YR, Sahgal A, Reynolds J, Chou D, Weber MH, Clarke MJ. A Systematic Review of Clinical Outcomes and Prognostic Factors for Patients Undergoing Surgery for Spinal Metastases Secondary to Breast Cancer. Global Spine J. 2016; 6(5): 482-96. PMID: 27433433 https://doi.org/10.1055/s-0035-1564807

14. Stacchiotti S, Palassini E, Sanfilippo R Vincenzi B, Arena MG, Bochicchio AM, De Rosa P, Nuzzo A, Turano S, Morosi C, Dei Tos AP, Pilotti S, Casali PG. Gemcitabine in advanced angiosarcoma: a retrospective case series analysis 
from the Italian Rare Cancer Network. Ann Oncol 2012; 23(2): 501-8. PMID: 21464156

https://doi.org/10.1093/annonc/mdr066

15. Stacchiotti S, Pedeutour F, Negri T Conca E, Marrari A, Palassini E, Collini P, Keslair F, Morosi C, Gronchi A, Pilotti S, Casali PG. Dermato fi brosarcoma protuberans- derived fi brosarcoma: clinical history, biological pro fi le and sensitivity to imatinib. Int J Cancer 2011; 129(7): 1761-72. PMID: 21128251

https://doi.org/10.1002/ijc.25826

16. Antman K, Crowley J, Balcerzak SP, Rivkin SE, Weiss GR, Elias A, Natale RB, Cooper RM, Barlogie B, Trump DL, et al. An intergroup phase III randomized study of doxorubicin and dacarbazine with or without ifosfamide and mesna in advanced soft tissue and bone sarcomas. J Clin Oncol. 1993; 11(7): 1276-85. PMID: 8315425 https://doi.org/10.1200/JCO.1993.11.7.1276

17. Rutkowski P, Van Glabbeke M, Rankin CJ Ruka W, Rubin BP, Debiec-Rychter M, Lazar A, Gelderblom H, Sciot R, Lopez-Terrada D, Hohenberger P, van Oosterom AT, Schuetze SM; European Organisation for Research and Treatment of Cancer Soft Tissue/Bone Sarcoma Group; Southwest Oncology Group. Imatinib mesylate in advanced dermato fi brosarcoma protuberans: pooled analysis of two phase II clinical trials. J Clin Oncol 2010; 28(10): 1772-9. PMID: 20194851

https://doi.org/10.1200/JCO.2009.25.7899
18. Rutkowski P1, Van Glabbeke M, Rankin CJ, Ruka W, Rubin BP, Debiec-Rychter M, Lazar A, Gelderblom H, Sciot R, Lopez-Terrada D, Hohenberger P, van Oosterom AT, Schuetze SM; European Organisation for Research and Treatment of Cancer Soft Tissue/Bone Sarcoma Group; Southwest Oncology Group. Imatinib mesylate in advanced dermatofibrosarcoma protuberans: pooled analysis of two phase II clinical trials. J Clin Oncol. 2010; 28(10): 1772-9. PMID: 20194851

19. Kummar S, Allen D, Monks A Polley EC, Hose CD, Ivy SP, Turkbey IB, Lawrence S, Kinders RJ, Choyke P, Simon R, Steinberg SM, Doroshow JH, Helman L. Cediranib for metastatic alveolar soft part sarcoma. J Clin Oncol 2013; 31(18): 2296-02. PMID: 23630200

https://doi.org/10.1200/JCO.2012.47.4288

20. Stacchiotti S, Tamborini E, Marrari A, Brich S, Rota SA, Orsenigo M, Crippa F, Morosi C, Gronchi A, Pierotti MA, Casali PG, Pilotti S. Response to sunitinib malate in advanced alveolar soft part sarcoma. Clin Cancer Res 2009; 15(3): 1096 - 104. PMID: 19188185

https://doi.org/10.1158/1078-0432.CCR-08-2050

21. Stacchiotti S, Negri T, Libertini M Palassini E, Marrari A, De Troia B, Gronchi A, Dei Tos AP, Morosi C, Messina A, Pilotti S, Casali PG. Sunitinib malate in solitary fi brous tumor (SFT). Ann Oncol 2012; 23(12): 3171-9. PMID: 22711763

https://doi.org/10.1093/annonc/mds143 